Изв. Крымск. Астрофиз. Обсерв. 117, № 1, 23-28 (2021) doi:10.31059/izcrao-vol117-iss1-pp23-28

\author{
УДК 523.9
}

\title{
Спектры пульсаций хромосферного излучения солнечных вспышек
}

\author{
Ю.А. Купряков ${ }^{1,2}$, А.Б. Горшков ${ }^{2}$, Л.К. Кашапова ${ }^{3}$ \\ 1 Astronomical Institute AS CR, Fričova 298, 25165 Ondřejov, Czech Republic \\ kupry@asu.cas.cz \\ 2 Астрономический институт им. П.К. Штернберга МГУ, Москва, 119234, Россия \\ gorshkov@sai.msu.ru \\ ${ }^{3}$ Институт солнечно-земной физики СО РАН, Иркутск, 664033, Россия \\ lkk@iszf.irk.ru
}

Поступила в редакцию 13 октября 2021 г.

\begin{abstract}
Аннотация. Мы представляем результаты анализа квазипериодических пульсаций (КПП) хромосферного излучения трех солнечных вспышек. Исследование основано на наблюдательных данных, которые были получены двумя наземными спектрографами: Multichannel Flare Spectrograph (MFS) и Horizontal-Sonnen-Forschungs-Anlage 2 (HSFA-2) обсерватории Ondřejov (Астрономический институт Чешской академии наук). Проведен анализ спектров мощности временных профилей, полученных как на основе спектрограмм, так и фильтрограмм. Проведено сравнение выявленных периодов с результатами анализа периодичности в рентгеновском и микроволновом диапазонах. Для тех событий, где позволила длительность наблюдений, обнаружен период, близкий к 5 минутам как по данным хромосферных наблюдений, так и по данным, полученным в рентгеновском и микроволновом диапазонах. Также были обнаружены периоды около 1.4-1.8 и 2-3 минут, которые одновременно проявлялись во всех исследованных диапазонах.
\end{abstract}

Ключевые слова: спектрограф, солнечные вспышки, квазипериодические осцилляции, хромосферное излучение

\section{1 Введение}

Квазипериодические пульсации (КПП) наблюдаются во временных профилях солнечных вспышек в широком диапазоне излучения - от микроволнового и УФ до излучения в хромосферных линиях и гамма-излучении. Продолжительность периодов КПП варьируется от субсекунд до десятков минут, что говорит о различной природе происхождения этих пульсаций. В настоящее время существует два основных класса механизмов. Первый связывает наблюдаемые пульсации с прямым воздействием магнитогидродинамических (МГД) волн, а второй - с повторяющимся процессом магнитного пересоединения (см. Kupriyanova et al., 2019, 2020). Часто механизмы двух классов сосуществуют и дополняют друг друга. Например, процесс магнитного пересоединения может быть инициирован МГД-волнами. Отсутствие или наличие КПП в нескольких спектральных диапазонах, изменение свойств от одного спектрального диапазона к другому позволяет не только уточнить вклад, но и провести диагностику свойств вспышечной плазмы.

Наша работа посвящена поиску КПП в хромосферном излучении солнечных вспышек, которое с одной стороны является наиболее наблюдаемым и характерным для этих событий. С другой стороны, излучение этой области солнечной атмосферы формируется под воздействием множества параметров, меняющихся в широком диапазоне, - температуры, плотности, движения вещества.

Для анализа мы выбрали три вспышки классов от С до M (SOL2011-04-22, SOL2013-05-19 и SOL2013-05-17), наблюдения которых были получены на обсерватории Чешской академии наук (Ondřejov) на спектрографах Multichannel-Flare-Spectrograph (MFS, 230 mm/13.5 m) и HorizontalSonnen-Forschungs-Anlage (HSFA-2, 500 mm/35 m). Также были использованы временные профили 
в рентгеновском диапазоне, полученные на Ramaty High Energy Solar Spectroscope Imager (RHESSI) (Lin et al., 2002), Gamma-Ray Burst Monitor (Fermi/GBM) of the Fermi Gamma-Ray Space Telescope (cм. Meegan et al., 2009) и наблюдения в микроволновом диапазоне, полученные на Radio Solar Telescope Network (RSTN).

\section{2 Наблюдения и обработка}

Нами были рассмотрены следующие солнечные вспышки:
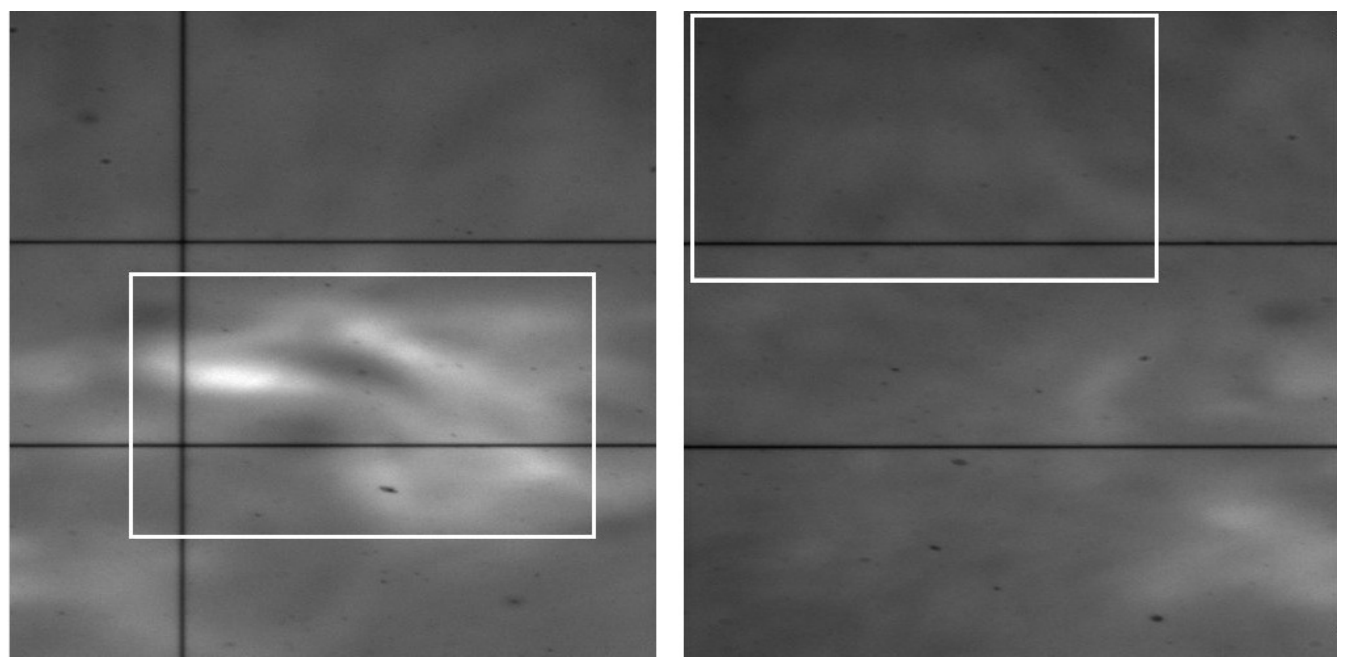

Рис. 1. Пример определения интеграла интенсивности со щели (фильтрограмма в линии $\left.\mathrm{H}_{\alpha}-\mathrm{SJ}\right)$ в активной (слева) и спокойной (справа) областях вспышки. Исследуемая область, в которой определяется интеграл, имеет размеры $200 \times 353$ пикселей
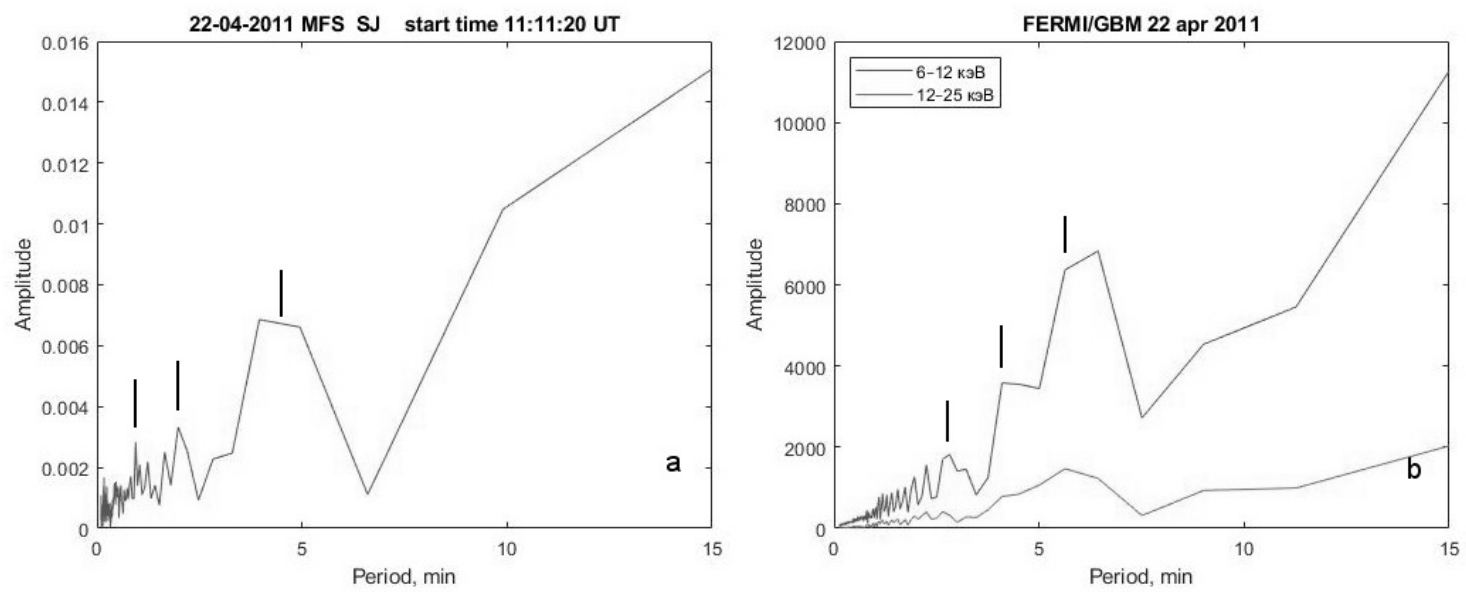

Рис. 2. а) Зависимость амплитуды мощности от периода Р. Отмеченные значения Р соответствуют $0.99,1.98$ и 4.7 мин. Обработанный временной интервал: 11:11:20-11:27:39 UT (продолжительность 16.3 минуты); b) На графике представлен спектр мощности данных, полученных FERMI. Отмеченные значения соответствуют периодам $2.97,3.96$ и 5 мин

а) Вспышка 22.04.2011 класса С2.6 произошла в активной области NOAA 11195 (начало 11:07, максимум 11:25, окончание 11:37 UT). Пример полученного изображения на HSFA представлен на рис. 1. Изображения записывались с интервалом 3 секунды. После калибровки снимков мы определяли интеграл интенсивности для активной и спокойной области. На рис. 2а представлена амплитуда мощности колебаний в зависимости от периода для обработанного интервала наблюдений 
11:11:20-11:27:39 UT, что соответствует области после максимума вспышки по данным GOES. При построении спектров мощности использовались процедуры быстрого фурье-преобразования с предварительным вычитанием тренда. На графике хорошо заметны несколько характерных максимумов с периодами колебаний $0.99,1.98$ и 4.7 минут.

Из других источников для этой вспышки нам удалось найти только данные FERMI/GBM. Они представлены на рис. $2 \mathrm{~b}$.

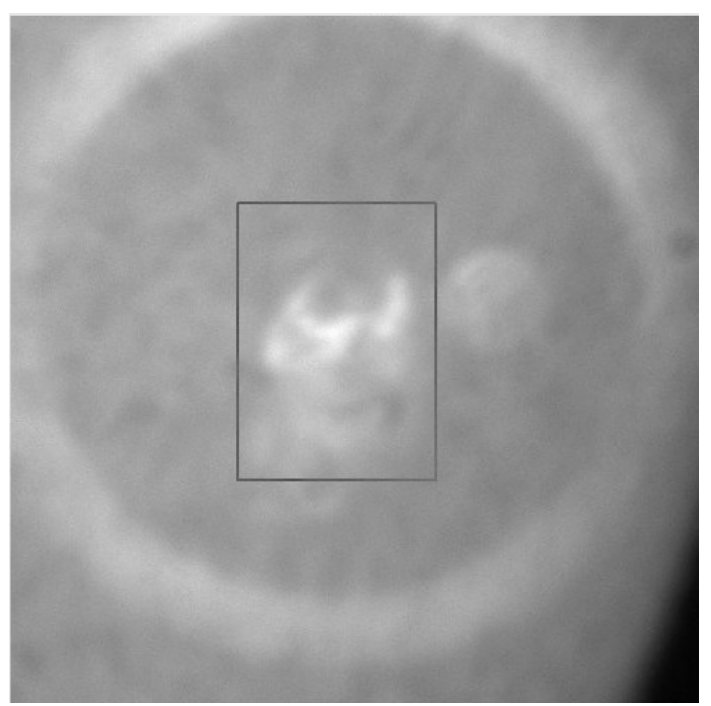

Рис. 3. Определение интеграла интенсивности для вспышки в активной области и участке спокойной хромосферы $(200 \times 143$ пикселей $)$. Белый круг вокруг активной области образован диафрагмой прибора
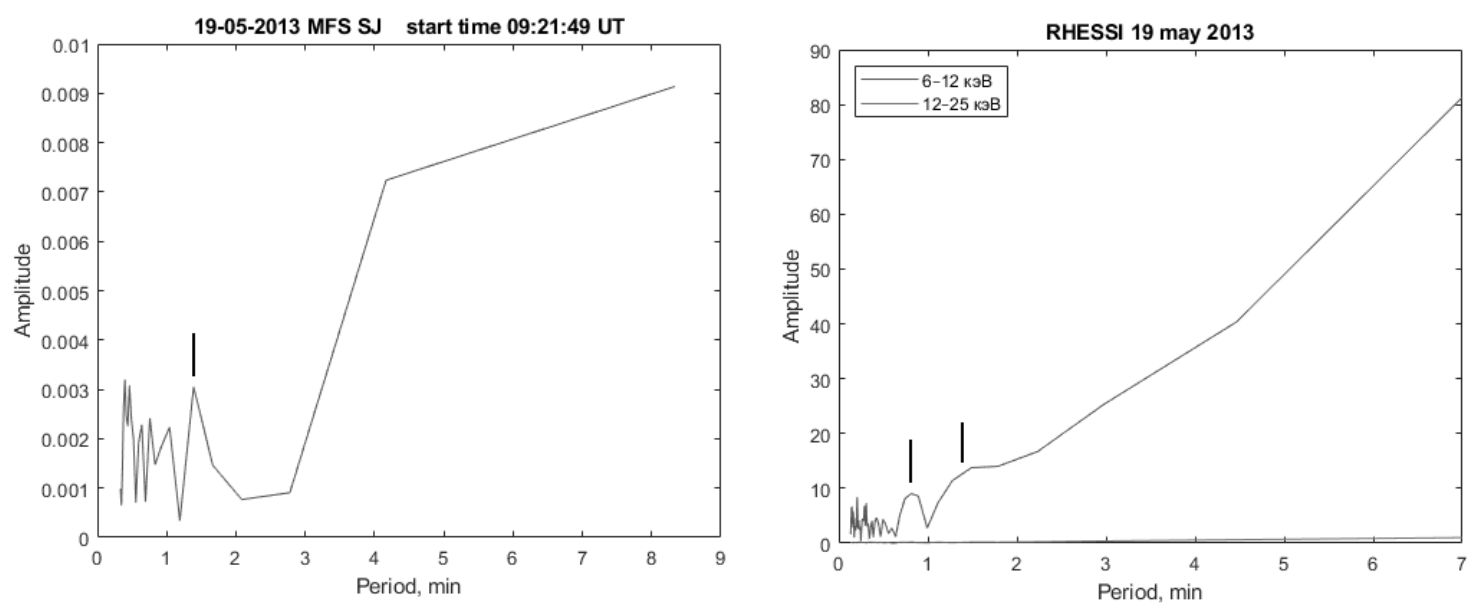

Рис. 4. а) Спектр мощности данных по кадрам SJ (продолжительность 7.8 минуты). Отмеченные значения соответствуют $\mathrm{P}=1.4$ мин; b) Спектр мощности RHESSI для 6-12 кэB, $\mathrm{P}=0.83$ и 1.4 мин

б) Вспышка 19.05.2013 класса C3.4 (начало 09:08, максимум 09:15, окончание 09:24 UT) наблюдалась в активной области NOAA 11750 на MFS. Данные записывались с интервалом 10 секунд. Временной интервал наблюдений 09:21:49-09:29:39 UT (продолжительность 7.8 минуты). На рис. 3 приведен снимок исследуемой области, а на рис. 4а - спектр мощности после обработки данных. Хорошо заметен максимум, соответствующий периоду колебаний 1.4 минуты.

Из внешних источников мы нашли только данные RHESSI. Спектр мощности для 6-12 кэB, построенный нами, показан на рис. 4b. Заметны периоды колебаний 0.83 и 1.4 минут.

в) И наконец, последняя вспышка, которую мы рассматривали, - 17.05.2013 класса М3.2 (начало 08:43, максимум 08:57, окончание 09:19 UT) в активной области NOAA 11748. Наблюдения проводились на спектрографах MFS и HSFA-2. 


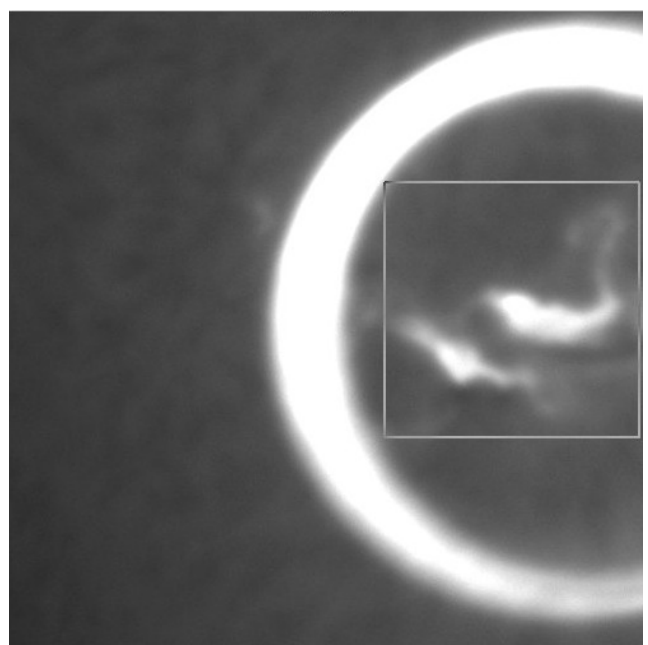

Рис. 5. Определение интегральной интенсивности вспышки 17.05.2013, MFS, М3.2 для интервала 08:42:43-09:27:09 UT. Белый круг - диафрагма прибора

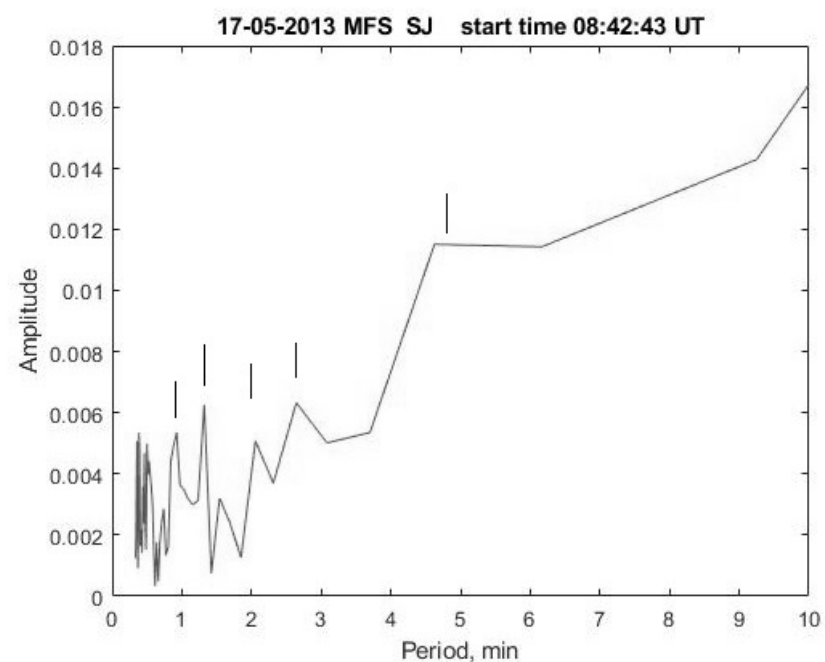

Рис. 6. Спектр мощности, отмеченные значения: 1.0, $1.2,2.0,2.8$ и 4.8 мин

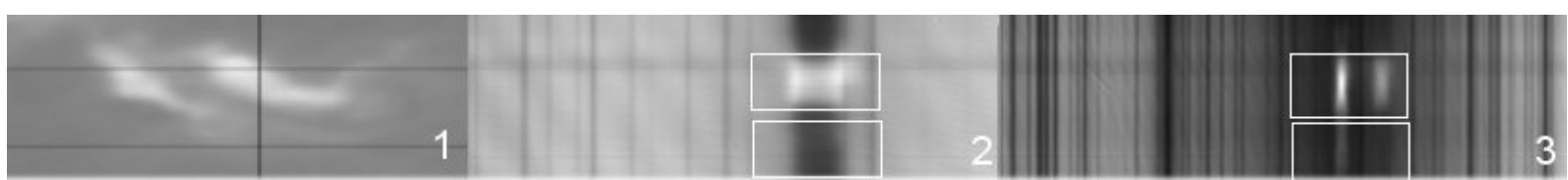

Рис. 7. Пример откалиброванных снимков SJ (1), Н $\alpha$ (2) и Н CaII (3), полученных на HSFA-2 17.05.2013 08:59:37.753 UT. Отмечены площадки, в которых определялась интегральная интенсивность в активной и спокойной области вспышки

Вышеописанным образом определялась интегральная интенсивность (рис. 5), и затем был построен спектр мощности. На графике рис. 6 можно выделить колебания с периодами 1.0, 1.2, 2.0, 2.8 и 4.8 минут.
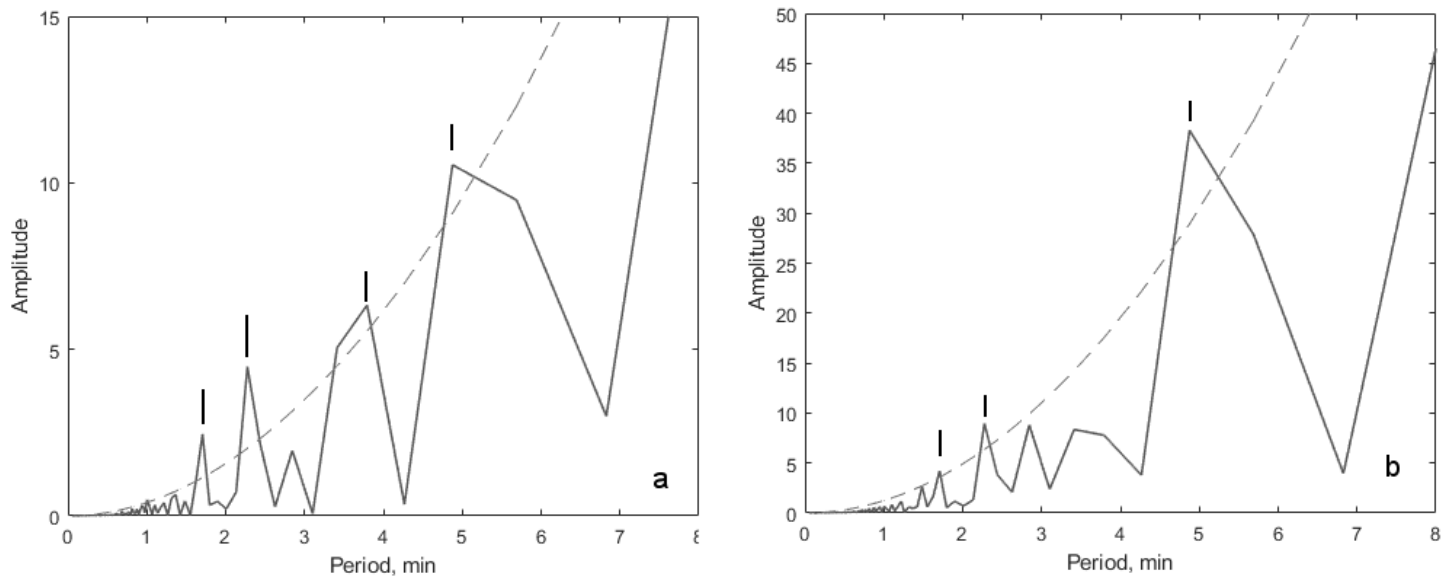

Рис. 8. Спектры мощности для интервала 08:41:53-09:01:36 UT (продолжительность 19.9 минут). Отмеченные значения: (a) $\mathrm{H}_{\alpha}-1.66,2.3,3.66,5$ мин; (b) H CaII - 1.83, 2.33, 5 мин. Пунктирной линией отмечен уровень значимости $95 \%$ 
Откалиброванные спектры, полученные на спектрографе HSFA-2 с интервалом 3 секунды, представлены на рис. 7. Результат построения спектров мощности - рис. 8.
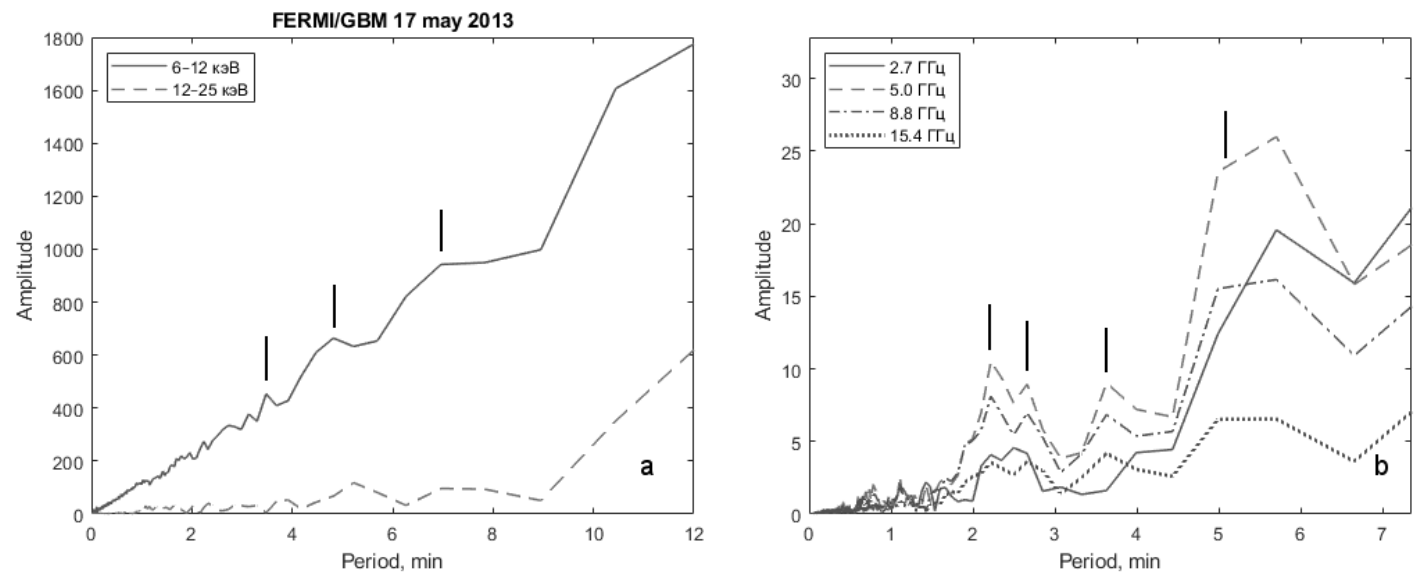

Рис. 9. а) Спектр мощности данных FERMI. Верхний график соответствует диапазону 6-12 кэВ. Отмеченные значения: 3.25, 5.0, 7.0 мин; b) Графики построены по данным RSTN. Отмеченные значения соответствуют колебаниям с периодами $2.23,2.84,3.67,5.0$ мин

Для данных FERMI 6-12 кэВ максимумы на графике (рис. 9а) соответствуют 3.25, 5.0 и 7.0 минутам, а для данных микроволнового излучения на частотах $2.7,5.0,8.8$ и 15.4 ГГц мы имеем колебания с частотами $2.23,2.84,3.67$ и 5.0 минут (рис. 9b).

\section{3 Выводы}

В ходе выполнения исследования были обработаны фильтрограммы (SJ) в линии $\mathrm{H}_{\alpha}$ и спектры в линиях $\mathrm{H}_{\alpha}$, H CaII. Получены временные профили для различных участков трех солнечных вспышек: 19 мая 2013 г. RHESSI; 17 мая 2013 г. и 22 апреля 2011 г. Данные хромосферных наблюдений были дополнены информацией об излучении в рентгеновском диапазоне (FERMI/GBM, RHESSI), а также для вспышки 17 мая 2013 г. наблюдениями в микроволновом диапазоне RSTN. Спектры мощности, построенные для исследованных событий, указывают на достаточно большой диапазон периодов колебаний хромосферного излучения от 1 до 5 минут. Для разных вспышек значения близких периодов незначительно варьируются. Сравнение полученных результатов для события 17 мая 2013 г. по данным двух спектрографов (рис. 6 и 8) дает близкие значения периодов по спектрам мощности колебаний. Отметим, что для событий, где продолжительность наблюдений была выше 15 минут, удалось уверенно выявить наличие периода около 5 минут. Также были обнаружены периоды около $1.4-1.8$ и 2-3 минут, которые наблюдались как в хромосферном излучении, так и в микроволновом и рентгеновском диапазонах. Предположительно, обнаруженные 5-минутные колебания имеют ту же природу, что и в работе Челпанов, Кобанов (2020), т. е. вспышка явилась модулятором уже существующих в хромосфере колебаний. Таким образом, полученные результаты можно использовать для изучения распространения МГД-волн в солнечных вспышках согласно методике, предложенной в этой работе. Отметим, что и другие обнаруженные периоды колебаний хромосферного излучения связаны с распространением МГД-волн, но их природа требует дополнительного исследования.

\section{Литература}

Челпанов А.А., Кобанов Н.И., 2020. Астрон. журн. Т. 97. № 4. С. 341. [Chelpanov A.A., Kobanov N.I., 2020. Astron. zhurn., vol. 97, no. 4, pp. 341-347. (In Russ.)]

Kupriyanova E.G., Kashapova L.K., Doorsselaere T.V., et. al. 2019. Mon. Not. Roy. Astron. Soc., vol. 483, pp. 5499-5507.

Kupriyanova E., Kolotkov D., Nakariakov V., and Kaufman A., 2020. Solar-Terrestrial Physics, vol. 6, pp. 3-23.

Lin R.P., Dennis B.R., Hurford G.J., et al., 2002. Solar Phys., vol. 210, pp.3-32.

Meegan C., Lichti G., Bhat P.N., et al., 2009. Astrophys. J., vol. 702, pp. 791-804. 


\title{
Solar flare pulsation spectra
}

Yu.A. Kupryakov ${ }^{1,2}$, A.B. Gorshkov ${ }^{2}$, L.K. Kashapova ${ }^{3}$

1 Astronomical Institute ASCR, Fričova 298, Ondřejov 251 65, Czech Republic

kupry@asu.cas.cz

2 Sternberg Astronomical Institute, Moscow State University, Universitetsky pr. 13, Moscow 119234, Russia gorshkov@sai.msu.ru

3 Institute of Solar-Terrestrial Physics SB RAS, Irkutsk 6640333, Russia lkk@iszf.irk.ru

\begin{abstract}
We present the results of the analysis of quasiperiodic pulsations (QPP) of chromospheric emission from three solar flares. The study is based on observational data obtained by two groundbased spectrographs: Multichannel Flare Spectrograph (MFS) and Horizontal-Sonnen-Forschungs-Anlage 2 (HSFA-2) of the Ondřejov Observatory (Astronomical Institute of the Czech Academy of Sciences). The analysis of the power spectra of the time profiles obtained both on the basis of spectrograms and filtergrams is carried out. The identified periods are compared with the results of periodicity analysis in the X-ray and microwave ranges. For those events where the duration of the observations allowed, a period close to 5 minutes was found both from the data of chromospheric observations and from the data obtained in the X-ray and microwave ranges. Also, periods were found in the region of 1.4-1.8 and 2-3 minutes, which simultaneously appeared in all studied ranges.
\end{abstract}

Key words: spectrograph, solar flares, quasiperiodic oscillations, chromospheric radiation 